\title{
Stochastic description of the dynamics of thrombo-embolus in an arterial compartment
}

\author{
Nzerem Francis Egenti*, Orumie Cynthia Ukamaka \\ Department of Mathematics \& Statistics, University of Port Harcourt, Nigeria \\ *Corresponding author E-mail: frankjournals@yahoo.com
}

Copyright (C) 2015 Nzerem Francis Egenti, Orumie Cynthia Ukamaka. This is an open access article distributed under the Creative Commons Attribution License, which permits unrestricted use, distribution, and reproduction in any medium, provided the original work is properly cited.

\begin{abstract}
Thrombosis, the formation or presence of blood clot in an arterial segment, most often admit the detachment of an embolus which flows antegrade. Embolism, the blockage of artery by an embolus, is a medical emergency. In a bid to gain an insight into its deleteriousness, we studied the diffusion of the embolus in an arterial compartment by means of Fokker-Planck equation, and the eventual occlusion of a site of the artery where the embolus was lodged. The probability density function of its spatial coordinate at any time was the hallmark of the diffusion process. Analytic solution of the emerging stochastic equation was sought. This study implicated thrombo-embolism in cardiovascular events.
\end{abstract}

Keywords: Artery; Diffusion Equation; Embolism; Fokker-Planck; Probability Density Function.

\section{Introduction}

Thrombosis is a pathogenic anomaly which is implicated in the deleterious effects of arterio-veinous embolism. Due to its tendency to obstruct flow, it may be implicated in arterial occlusion which induces sudden acute pain with numbness, tingling, pallor, weakness and coldness [1]. The direct effect of these include: cyanosis, mottling, retardation of motor function. A large thrombus could disintegrate to generate emboli. Emboli are foreign masses (particles) which flow with the fluid body (the drift process). Arterial emboli originate from the heart. If they are not dissolved,they may travel to the brain (which may cause a stroke) or in the heart (which may cause a heart attack), spleen, vessels and the lower extremities. Less common sites include the kidneys, intestines, and eyes. When they get lodged at any of such places they obstruct flow. The upstream motion is accompanied by diffusion of the particles. Thus, there exists a two-phase flow consisting of whole blood as the medium and the emboli as particles in anterograde motion.

The description of motion of an embolus is modeled by a stochastic process (SP) mostly associated with the diffusion component. This follows from the Brownian motion that characterizes the micro-particulate nature of an embolus involved in the diffusion process. The most suiting of equations for Brownian motion with different degrees of freedom is Langevin equation. This equation has appeared in varieties of literature [2], [3], [4], [5].It is a stochastic differential equation driven by an additive Gaussian white noise. Interestingly, there exists relationship between Langevin equation and Fokker-Planck equation in describing fluctuations associated with the particle motion [6]. Fokker-Planck equation is a second order partial differential equation describing the time evolution of the probability density function of a SP. Besides, the Ornstein-Uhlenbeck process (OUP) [7] derives from Langevin equation. Ole et al [5] studied the existence and properties of stationary solutions to Langevin equation driven by a noise process. Langevin equation, FokkerPlanck equation and the OUP altogether hold well in the description of the motion of an arterial embolus.

In what seems to be rather disturbing, the configuration(s) other than a potential well in which particle motion is described may not have been given substantial attention in literature. In real world, including a physiological setting associated with the present study, the domain of influence is necessary. We therefore suggested motion in a circular cylinder. This seems reasonable by virtue of cylindrical nature of an arterial segment. However, the cylinder is treated as a boundary. This is to say that the arterial motion in response to blood flow is not taken into account. In the present 
case, such a response is, to say the least, unnecessary. We considered the stationary solution of the defining stochastic differential equation. This is physiologically contextual since blood flow tends to be steady as arterial diameter decreases. This account for the near steady state flow in capillaries of diameters less than $8 \mu \mathrm{m}$. The motion of blood is pressure-driven; in the capillaries pressure is not purely time dependent. It is largely dependent on the radius of the capillary [8]. We therefore conceived of stationary solution of the defining stochastic differential equation. Any region of arterial occlusion is marked by almost no flow; total occlusion amounts to total flow stoppage. Necrotic parts of the body sometimes present with stationary flow, induced possibly by arterial embolism of the connecting segment.

\section{Governing stochastic equation}

The one -dimensional anterograde motion of a particle in generalized medium is of the for

$m \frac{d^{2} x}{d t^{2}}=-\lambda \frac{d x}{d t}-\frac{d V}{d x}+\Phi(\mathrm{t})$

where

$\mathrm{m}$ is the mass of the particle,

The first term on the right -hand side (r.h.s) is due to fluid viscosity,

$\lambda$ is the friction constant,

The second term on the r.h.s is the interaction of the particle with external forces,

$\mathrm{V}(\mathrm{x})$ is a potential, and the third term on the r.h.s. $\Phi(\mathrm{t})$ is a random force due to particle collision with fluid molecules.

The dynamics of the particle requires the initial position and velocity of the particle, and the statistical description of the random force $\Phi(\mathrm{t})$. We give the average position of the particle as $\langle x(t)\rangle$ and that of velocity as $\langle v(t)\rangle$ at time $\mathrm{t}$ conditional on $\mathrm{x}(0)=\mathrm{x}_{0}$ and $\mathrm{v}(0)=\mathrm{v}_{0}$. The fluctuating force $\Phi(\mathrm{t})$ is such that

$$
\langle\Phi(t)\rangle=0, \quad\left\langle\Phi(t) \Phi\left(t^{\prime}\right)\right\rangle=\sigma^{2} \delta\left(t-t^{\prime}\right)
$$

The autocorrelation function $\left\langle\Phi(t) \Phi\left(t^{\prime}\right)\right\rangle$ is a Dirac delta function, with $\sigma$ being the weight of the delta-function. The presence of the acceleration term $m \frac{d^{2} x}{d t^{2}}$ makes equation (2.1) non-Markovian (see [9], [10]). Indeed, determining the current state of the system from memory of entire history of the state other than the immediate past is not necessary. Schieber and Ottinger [11] explained why the said term may be neglected. If we assume the absence of external forces and write $d x / d t=v$, we get the Langevin equation

$$
\frac{d v}{d t}=-\gamma+F(t) ; \quad v(0)=v_{0}
$$

where $\gamma=\lambda / \mathrm{m}, \mathrm{F}(\mathrm{t})=\Phi(\mathrm{t}) / \mathrm{m}$ and $\mathrm{v}$ is the particle velocity. Thus,

$$
F(t)=0, \text { And } F(t) F\left(t^{\prime}\right)=\frac{\sigma^{2}}{2 m^{2}}\left(t-t^{\prime}\right)
$$

In equation (2.3) the Gaussian white noise $\mathrm{F}(\mathrm{t})$, is a formal derivative of a zero mean Weiner process $\mathrm{W}(\mathrm{t})$. For the fact that $\mathrm{W}$ is nowhere differentiable, $\mathrm{F}$ is now a distribution rather than a function [12].

Uninterestingly our Langevin equation (2.3) does not contain the spatial term x explicitly, owing to the need to dispense with the acceleration term in equation (2.1). No cause for alarm! We recourse to adiabatic elimination of the velocity process by means of Smoluchowski equation for the position x. Having done this, we write the evolution equation of the deterministic differential equation as

$$
\frac{d x}{d t}=\frac{F(x(t))}{\gamma m}
$$

with the corresponding stochastic equation generated as 
$\frac{\partial}{\partial t} p\left(x, t \mid x_{0}, t_{o}\right)=-\frac{\partial}{\partial x}\left[D^{1} p\left(x, t \mid x_{o}, t_{o}\right)\right]+D^{2} \frac{\partial^{2}}{\partial x^{2}} p\left(x, t \mid x_{o}, t_{o}\right)$

In the above equation $D^{1}=\frac{F(x)}{\lambda m}$ is the drift process while $D^{2}=\frac{\sigma^{2}}{2 \gamma^{2} m^{2}}$ is the diffusion process involved in the particle motion. The stochastic nature underscores the invitation of the probability density function $\mathrm{p}(\mathrm{x}, \mathrm{t})$. The FokkerPlanck equation (2.6) for the variable $\mathrm{x}$ is known as the Smoluchowski equation.

\section{Particle motion in the cylinder}

In this section we shall consider the motion of an embolus in an arterial segment. The artery is considered as a circular cylinder. It is instructive to note that the arterial wall is treated as a natural boundary; hence the arterial motion in response to the fluid flow is not taken into account. Specifically, we analyze the "uncorrelated random walk" (Brownian motion) of the particle associated with diffusion. The analogous Fick's equation of diffusion

$$
\frac{\partial c(x, t)}{\partial t}=D \frac{\partial^{2} c(x, t)}{\partial x^{2}}
$$

where $\mathrm{c}$ may be the concentration of the particle, and $\mathrm{D}$ a constant is evident in (2.6) when $\mathrm{D}^{1}=0$.

Suppose the particle in question is allowed to diffuse in a non-tapering segment of the cylinder of radius $\rho$ and axis $\mathrm{z} \epsilon$ $\left[0, \mathrm{~L}_{0}\right]$. The governing diffusion equation may be given by

$$
\frac{\partial}{\partial t} p\left(x, y, z, t \mid x_{0}, y_{0}, z_{0}, t_{o}\right)=-\bar{D}\left(\frac{\partial^{2}}{\partial x^{2}}+\frac{\partial^{2}}{\partial y^{2}}\right) i p\left(x, y, z, t \mid x_{0}, y_{0}, z_{0}, t_{o}\right)+\hat{D} \frac{\partial^{2}}{\partial z^{2}} p\left(x, y, z, t \mid x_{0}, y_{0}, z_{0}, t_{o}\right)
$$

where $\bar{D} \neq \hat{D}$. A consideration of axisymmetricity in relation to the cylindrical coordinates (r, $\phi, \mathrm{z})$ gives

$$
\partial_{t} p\left(r, z, t \mid r_{0}, z_{0}, t_{0}\right)=\bar{D} \frac{1}{r} \frac{\partial}{\partial r}\left(r \frac{\partial p}{\partial r}\right)+\hat{D} \partial_{z}^{2} p\left(r, z, t \mid r_{0}, z_{0}, t_{0}\right)
$$

We shall seek a stationary solution of equation (3.3), with the stipulation $\partial_{\mathrm{t}} \mathrm{p}=0$. Thus,

$$
\frac{\partial^{2}}{\partial r^{2}} p_{s}\left(r, z \mid r_{0}, z_{0}, t_{0}\right)+\frac{1}{r} \frac{\partial}{\partial r} p_{s}\left(r, z \mid r_{0}, z_{0}, t_{0}\right)+\eta^{2} \frac{\partial^{2}}{\partial z^{2}} p_{s}\left(r, z \mid r_{0}, z_{0}, t_{0}\right)=0
$$

Where:

$$
\eta^{2}=\frac{\bar{D}}{\hat{D}}, \quad \bar{D}>0, \quad \hat{D}>0
$$

For a reflecting wall we get the boundary condition (b. c.)

$$
\left.\frac{\partial}{\partial r} p_{s}\left(r, z \mid r_{0}, z_{0}, t_{0}\right)\right|_{r=\rho}=0
$$

We now consider when the embolus adheres to the arterial wall. This is to say that $\mathrm{z}=0, \mathrm{r} \leq \mathrm{R}$ is an absorbing boundary, so $p_{S}\left(r, 0 \mid r_{0}, z_{0}, t_{0}\right)=0$ while $\mathrm{z}=\mathrm{L}_{0}$ has a constant value $\mathrm{p}_{\mathrm{k}}$. By using equation (3.4) and by application of separation of variables we may write the stationary solution in the form

$$
p_{S}\left(r, z \mid r_{0}, z_{0}, t_{0}\right)=R(r) \mathrm{Z}(\mathrm{z})
$$


The adherence to the arterial wall may be seen as regarding that portion of the wall as stenosed. Therefore, the radius of the occluded region of the artery is given in the form [13]

$$
r_{0}=R\left[1-\frac{h}{2 R}\left(1+\cos \frac{\pi z_{0}}{\bar{h}}\right)\right],
$$

where

$\mathrm{R}=$ radius of artery at the non-stenosed regions;

$\mathrm{r}_{0}=$ radius at the stenosed regions;

$\mathrm{h}=$ height of the stenosis;

$\bar{h}=$ width of the stenosis;

$\mathrm{z}_{0}=$ location of any point of interest on the axis.

The magnitude of occlusion of the artery at the point $\mathrm{z}=\mathrm{z}_{0}$ depends on the stenosis parameters. It is this occlusion that invigorates the pathogenesis of arterial thrombo-embolism.

Using equation (3.7) in equation (3.4) we get

$$
R^{\prime \prime} Z+r^{-1} R^{\prime} Z+\eta R Z^{\prime \prime}=0 .
$$

By separation of variables we get

$$
\frac{R^{\prime \prime}}{R}+r^{-1} \frac{R^{\prime}}{R}=-\eta^{2} \frac{Z^{\prime \prime}}{Z}=-\mu^{2} .
$$

The radial part takes the form

$$
r^{2} R^{\prime \prime}+r R^{\prime}+r^{2} \mu R=0
$$

with the general solution of the form

$$
R(r)=A_{1} J_{0}(\mu r)+A_{2} H_{0}(\mu r),
$$

where $\mathrm{J}_{0}$ and $\mathrm{H}_{0}$ are Bessel functions of zero order. At $\mathrm{H}_{0}(0)=-\infty$ and $\mathrm{R}(0)<\infty$, we put $\mathrm{A}_{2}=0$ to get

$$
R(r)=A_{1} J_{0}(\mu r) .
$$

For no-flux condition at the surface, the values of $\mu$ that satisfy $A_{2} \neq 0$ is given by the condition

$$
\left.\frac{d J_{0}(x)}{d x}\right|_{x=\mu_{m} \rho}=0 .
$$

This, we apply to get

$$
R(r)=A_{2} J_{0}\left(\mu_{m} r\right)
$$

For a given value of $\mu_{\mathrm{m}}$ we solve for $Z(z)$ :

$$
Z^{\prime \prime}-\frac{\mu_{m}^{2}}{\eta^{2}} Z=0
$$

The general solution of the above equation is 
$Z_{m}(z)=B_{1} \sinh \left(\frac{\mu_{m}}{\eta} z\right)+B_{2} \cosh \left(\frac{\mu_{m}}{\eta} z\right)$.

The absorbing b.c. at $z=0$ gives $Z(0)=0$. Thus,

$Z(z)=B_{1} \sinh \left(\frac{\mu_{m}}{\eta} z\right)$

For fixed $\mu_{m}$ we get

$p_{s m}(r, z)=B_{1 m} \sinh \left(\frac{\mu_{m}}{\eta} z\right) J_{0}\left(\mu_{m} r\right), \mathrm{m}=1,2 \ldots$

Thus,

$p_{s}(r, z)=\sum_{m=1}^{\infty} B_{1 m} \sinh \left(\frac{\mu_{m}}{\eta} z\right) J_{0}\left(\mu_{m} r\right)$.

With $p\left(z=L_{0}, r\right)=p_{c}$ we get

$p_{c}=\sum_{m=1}^{\infty} B_{1 m} \sinh \left(\frac{\mu_{m}}{\eta} L_{0}\right) J_{0}\left(\mu_{m} r\right)$

The quantity $B_{1 m}$, by virtue of properties of Bessel function and orthogonality property in [0,1], yields

$$
B_{1 m}=\frac{2 p_{c}}{\mu_{m} J_{1}\left(\mu_{m}\right) \sinh \left(\frac{\mu_{m}}{\eta} L_{0}\right)}
$$

We therefore write the steady state solution as

$$
p_{s}(r, z)=\sum_{m=1}^{\infty} \frac{2 p_{k}}{\mu_{m} J_{1}\left(\mu_{m}\right) \sinh \left(\frac{\mu_{m}}{\eta} L_{0}\right)} \sinh \left(\frac{\mu_{m}}{\eta} z\right) J_{0}\left(\mu_{m} r\right)
$$

\section{Discussions}

We have studied the stochastic dynamics of an arterial embolus antegrade to the flowing blood. Generalized Langevin differential equation models the SP involved in random walks in an unspecified region. The motion of an embolus with the flowing blood is in the arterial segment. The artery is modeled as a circular cylinder. The random collision of the embolus with fluid body and particles gives room for uncertainties which time may find difficult to explain. But time evolution of the probability density function (pdf) of the SP is of essence; Focker-Planck equation, derivable from Langevin differential equation was used in obtaining this. The location of a particle undergoing a random walk in space is in the least deterministic. Thus, the pdf gives a clue to the tendency of finding the position of the embolus.

Physiological perspective of the study indicates that arterial embolism is a liability. Occlusion, whether partial or total, is an ill wind; it is instrumental in many debilitating conditions, including strokes and infarctions. However, an embolus may travel innocuously upstream, but it may be eventually pernicious when it is lodged at any arterial site. Fortunately, medical interventions that involve regimens of anti-clot agents like anti-platelets (such as aspirin, clopidogrel and ticlopidine) or anticoagulants (such as heparin, warfarin) are effectual in the prevention of thrombosis.

Equation (3.8) shows the radius of an occluded region of an arterial segment $z \in\left(0, L_{0}\right)$. We consider where the occluded region $r_{0}$ is at maximum. Suppose $h \rightarrow R$; equation (3.8) gives 
$r_{0}=\frac{R}{2}\left(1-\cos \frac{\pi z_{0}}{\bar{h}}\right), \quad \bar{h} \neq 0$.

The minimum and maximum values of $\cos \frac{\pi z}{\bar{h}}$ occur at -1 and +1 respectively. $\mathrm{r}_{0}$ attains a maximum value when we choose $\bar{h}$ such that $\cos \frac{\pi z}{\bar{h}}$ is at minimum (i.e. at -1 ). Thus, $\mathrm{r}_{0}=\mathrm{R}$. This shows that the entire radius is occluded. Flow therefore is extremely retarded. At $\cos \frac{\pi z_{0}}{\bar{h}}=+1$. Observe that $\cos \frac{\pi z_{0}}{\bar{h}}=+1$ means $\mathrm{z}_{0}=0$ since $\bar{h} \neq 0$. This is open to two considerations:

(i) $r_{0}=0$ corresponds to the region of no-occlusion region.

(ii) If occlusion exists and $z_{0}=0 \rightarrow r_{0}=0$; then it is at the origin (beginning) of the arterial length. Since $r_{0}=0$ means a radius of magnitude zero, we ask: What is the mathematical meaning of zero radiuses? It may well stand for a point [14], [15]. Thus, the occlusion at the origin means $\bar{h}$ is considerably small. We note that there are two conditions upon which $\mathrm{r}_{0}$ can be zero; when $\mathrm{R}=0$ or $\mathrm{z}_{0}=0$. But $\mathrm{R}=0$ is inadmissible; therefore the first consideration only holds if $\mathrm{z}_{0}$ is inside the cylinder. We surmise that $\mathrm{z}_{0}=0 \rightarrow \mathrm{r}_{0}=0$ is a point of early formation of a thrombus at the origin.

Consider equation (3.23) once more. Let $r=r_{0}, \quad z=z_{0}$. Then we plant (3.8) in (3.23) to get

$$
p_{S}\left(r,\left.z \quad\right|_{r=r_{O}, z=z_{0}}\right)=\sum_{m=1}^{\infty} \frac{2 p_{k}}{\mu_{m} J_{1}\left(\mu_{m}\right) \sinh \left(\frac{\mu_{m}}{\eta} L_{0}\right)} \sinh \left(\frac{\mu_{m}}{\eta} z_{0}\right) J_{0}\left(\mu_{m} r_{0}\right)
$$

The above equation therefore gives the probability of the radial and axial coordinate of the embolus at $\mathrm{r}_{0}$ and $\mathrm{z}_{0}$. From the analysis, it is instructive that any medical intervention must target a reduction of $h$. A consistent reduction amounts to liquefaction of the embolus. It is noteworthy that such reduction increases $\bar{h}$. However, this is admissible since it will eventually lead to the evanescence of the embolus.

\section{References}

[1] J.N. Mazumdar, Biofluid Mechanics, World scientific publishing co. Pte ltd., 1992.

[2] P.A. Anarak,i., The exact Solution of Fokker Planck Equation for Brownian Motion.,The African Review of Physics 7: 0021 (2012) $215-218$.

[3] R.Guo and J.Du, Power-law behaviours from the two-variable Langevin equation: Ito's and Stratonovich's Fokker-Planck equations, stacks.iop.org/JSTAT/2013/P02015; doi:10.1088/1742-5468/2013/02/P02015.Accessed December7, 2014.

[4] Donev, A. (2013) Langevin Equations for Fluctuating Hydrodynamics, http;//cism.nyu.edu/ donev/Teaching/CoarseGrainingFall2013/Lectur... Accessed December 8, 2014.

[5] O. E. Ole, E. Barndorff-Nielsen and B. A. Andreas, Quasi Ornstein-Uhlenbeck processes, http://dx.doi.org/10.3150/10-BEJ311.

[6] Chapman-Kolmogorov for equation continuous $\frac{\text { paths: }}{\text { Fokker-Plank }}$ http://www.pd.infn.it/ orlandin/fisica_sis_comp/fokker_plank.pdf. Accessed December 7, 2014.

[7] G.E.Uhlenbeck, and 1. Ornstein Phys Rev 36 (1930) 823. http://dx.doi.org/10.1103/PhysRev.36.823.

[8] G.C.E. Mbah, and H.O. Adagba, Flow through a constricted capillary, Jour. Of Mathematical Sciences, Vol. 21, No. 2: (2010) 115-126.

[9] D.T. Gillespie, Markov Processes, Academic Press, San Diego, 1992.

[10] A.J. McKane, Stochastic Process, http://www.theory.physics.mancheter.ac.uk Accessed December10, 2014.

[11] H.C. Schieber, and J.D. Ottinger, The effects of bead inertia on the Rouse model. The Journal of Chemical Physics, 89(11) (1988) 69-72 http://dx.doi.org/10.1063/1.455323.

[12] P. Holmes, J.L. Lumley and G. Berkooz, Turbulence, Coherent Structures and Symmetry, Cambridge University Press, (1996) http://dx.doi.org/10.1017/CBO9780511622700.

[13] D.F. Young, Effects of a time-dependent stenosis on flow through a tube, J. Engr.Ind.90 (1968) 248-254 http://dx.doi.org/10.1115/1.3604621.

[14] S. Schwartzman, The Words of Mathematics: An Etymological Dictionary of Mathematical Terms Used in English, Spectrum, 1996.

[15] What Is Point? http://www.cut-the-knot.org/WhatIs/WhatIsPoint.shtml Accessed December10, 2014. 